\title{
Propofol Suppresses Gastric Cancer Progression by Regulating circPDSSI/miR-I324/SOX4 Axis
}

\author{
Leyi Liu' \\ Ting Dong ${ }^{2}$ \\ Jun Sheng ${ }^{3}$
}

'Department of Anesthesiology, Wuhu Hospital of Traditional Chinese Medicine, Wuhu, 241000, People's Republic of China; ${ }^{2}$ Yijishan Hospital, First Affiliated Hospital of Wannan Medical College, Wuhu, 24I00I, People's Republic of China; ${ }^{3}$ Department of Oncology, Wuhu Hospital of Traditional Chinese Medicine, Wuhu, 241000, People's Republic of China

Correspondence: Jun Sheng

Department of Oncology, Wuhu Hospital of Traditional Chinese Medicine, No. 430, jiuhua South Road, Yijiang District, Wuhu, 241000, People's Republic of China Email sj6785@I26.com
Background: Propofol is a common intravenous anesthetic that exerts an antitumor role in human cancers. Circular RNAs (circRNAs) play crucial roles in the progression of various cancers. However, the relationship between propofol and circRNA decaprenyl diphosphate synthase subunit 1 (circPDSS1) in gastric cancer (GC) remains unclear.

Methods: Cell proliferation was evaluated by Cell Counting Kit-8 (CCK-8), colony formation, and 5-ethynyl-2'-deoxyuridine (EdU) assays. Cell migration and invasion were assessed by transwell assay. Cell apoptosis was determined by flow cytometry. All protein levels were detected by Western blot assay. The expression levels of circPDSS1, microRNA-1324 (miR1324), and SRY-box transcription factor 4 (SOX4) mRNA were determined by quantitative real-time PCR (qRT-PCR). The interaction between miR-1324 and circPDSS1 or SOX4 was confirmed by dual-luciferase reporter and RNA pull-down assays. The mice xenograft model was established to investigate the role of propofol and circPDSS1 in vivo.

Results: Propofol inhibited cell proliferation, migration and invasion and induced apoptosis in GC cells, which could be reversed by upregulating circPDSS1. MiR-1324 was a target of circPDSS1, and circPDSS1 promoted cell proliferation, migration and invasion and reduced apoptosis in propofol-treated cells by sponging miR-1324. Moreover, SOX4 was a direct target of miR-1324, and miR-1324 exerted anticancer role by targeting SOX4 in propofoltreated cells. CircPDSS1 acted as a sponge of miR-1324 to regulate SOX4 expression. Additionally, circPDSS1 overexpression weakened the anticancer role of propofol in vivo. Conclusion: Propofol exerted anticancer role in GC through regulating circPDSS1/miR1324/SOX4 axis, indicating that propofol might be an effective therapeutic medicine for GC treatment.

Keywords: gastric cancer, propofol, circPDSS1, miR-1324, SOX4

\section{Introduction}

Gastric cancer (GC) is the second leading cause of cancer-related mortality worldwide with an estimated over 769,000 deaths in 2018. ${ }^{1}$ Although outstanding advances have been made in therapeutic methods, the 5-year overall survival rate is less than $30 \%$ in most countries because of frequent tumor metastasis and recurrence. ${ }^{2}$ Therefore, it is of great significance to understand and study the molecular mechanisms of GC pathogenesis to improve the survival of GC patients.

Propofol is one of the commonly used intravenous anesthetics in clinical operation, characterized by short action, rapid recover, and little side effect. ${ }^{3}$ In addition, its multiple anesthetic advantages, propofol also has multiple non-anesthetic effects. ${ }^{4}$ Many studies have reported that propofol can exert antitumor function via different molecular mechanisms in diverse cancers, such as bladder carcinoma, ${ }^{5}$ lung cancer, ${ }^{6}$ 
pancreatic cancer, ${ }^{7}$ and colorectal cancer. ${ }^{8}$ Moreover, propofol also suppresses GC cell proliferation and metastasis. ${ }^{9}$ However, the detailed molecular mechanism of propofol in $\mathrm{GC}$ is needed to be further explored.

As a novel type of non-coding RNAs (ncRNAs), circular RNAs (circRNAs) possess covalently closed-loop RNA structures. ${ }^{10}$ Many reports have confirmed the critical importance of circRNAs in human diseases, including cancers. ${ }^{11,12}$ With rapid advances in bioinformatics technologies and high-throughput sequencing, some circRNAs have been found to be involved in the development of GC. ${ }^{13-15}$ CircRNA decaprenyl diphosphate synthase subunit 1 (circPDSS1, also known as hsa_circ_0093398) is derived from PDSS1 gene and located at chr10:2699421427024508. CircPDSS1 has been reported to accelerate GC progression. ${ }^{16}$ However, whether circPDSS1 was involved in propofol-mediated functions in GC is still unclear.

It is well known that circRNAs can function as sponges for microRNAs (miRNAs) via competitively binding to miRNA response elements, thereby modulating the expression of downstream target genes. ${ }^{17,18}$ MiRNAs are small ncRNAs that are involved in cancer progression as a suppressors or promoters. ${ }^{19}$ MiR-1324 has been reported to play tumor-suppressing miRNA in GC. ${ }^{20}$ SRY-box transcription factor 4 (SOX4), an essential developmental transcription factor, has been suggested to have vital roles in the embryonic development and cell fate decision. ${ }^{21}$ Moreover, increased expression of SOX4 has been observed in many cancers, including GC. ${ }^{22-24}$ Interestingly, online bioinformatics database showed that both circPDSS 1 and SOX4 had complementary binding sequence for miR-1324. Therefore, we hypothesized that propofol might exert anticancer role in $\mathrm{GC}$ via modulating circPDSS1/miR-1324/SOX4 axis.

In this paper, we investigated the role of propofol in $\mathrm{GC}$ and explored the association between propofol and circPDSS1/miR-1324/SOX4. We aimed to offer a new insight into the use of anesthetics for the treatment of GC.

\section{Materials and Methods Specimen Collection}

$\mathrm{GC}$ tissues $(\mathrm{n}=35)$ and adjacent normal tissues $(\mathrm{n}=35)$ were obtained from GC patients who underwent surgery at Wuhu Hospital of Traditional Chinese Medicine. Tissue specimens were harvested at surgery, promptly snapfrozen in liquid nitrogen and then stored at $-80^{\circ} \mathrm{C}$. Written informed consent was obtained from all patients.
This study was approved by the Research Ethics Committee of Wuhu Hospital of Traditional Chinese Medicine.

\section{Cell Culture and Transfection}

GC cells (HGC-27 and AGS) were purchased from BeNa Culture Collection (Beijing, China) and cultured in Dulbecco's Modified Eagle Medium (Invitrogen, Carlsbad, CA, USA) that contained $10 \%$ fetal bovine serum (FBS; Invitrogen), $100 \mu / \mathrm{mL}$ penicillin and $100 \mathrm{mg} / \mathrm{mL}$ streptomycin (Gibco, Carlsbad, CA, USA) in incubator at $37^{\circ} \mathrm{C}$ with $5 \% \mathrm{CO}_{2}$. Propofol was acquired from Sigma-Aldrich (St. Louis, MO, USA) and added to cells in a concentration gradient.

CircPDSS1 overexpression vector (circPDSS1) and matched control (Vector), small interfering RNA (siRNA) against circPDSS1 (si-circPDSS1) and matched control (si-NC), miR-1324 mimic or inhibitor (miR-1324 or in-miR-1324) and matched control (miR-NC or in-miR$\mathrm{NC}$ ), SOX4-overexpressing plasmid (SOX4) and matched control (pcDNA) were all purchased from RiboBio (Guangzhou, China). Cells were transfected with the above oligonucleotide (50 $\mathrm{nM}$ miRNA mimic/inhibitor and $20 \mathrm{nM}$ siRNA) or plasmid $(2 \mu \mathrm{g})$ using Lipofectamine 3000 (Invitrogen) when cells reached 60$70 \%$ confluence.

\section{Cell Viability Assay}

Cell Counting Kit-8 (CCK-8) assay was used to analyze cell viability. In short, cell suspension $\left(100 \mu \mathrm{L}, 2 \times 10^{3}\right.$ cells/well) were inoculated in 96-well plates. CCK-8 (10 $\mu \mathrm{L}$, Bimake, Shanghai, China) was placed into each well after respective treatment. After incubation for $2-3 \mathrm{~h}$, the absorbance at $450 \mathrm{~nm}$ was examined using a microplate reader (Bio-Rad, Hercules, CA, USA). All experiments were repeated three times.

\section{Colony Formation Assay}

Cells ( 150 cells/well) were inoculated in a 6-well plate and the medium was updated every 2-3 day, and then cultured for about 14 days to form colonies. Afterwards, these colonies were fixed in 4\% paraformaldehyde (Sangon Biotech, Shanghai, China) for $15 \mathrm{~min}$, followed by staining with $0.1 \%$ crystal violet (Beyotime, Jiangsu, China) for half an hour. Thereafter, excess crystal violet was washed using phosphate buffered saline (PBS; Beyotime), and the colonies were counted and analyzed. All experiments were repeated three times. 


\section{5-Ethynyl-2'-Deoxyuridine (EdU) Assay}

Cell proliferation and DNA synthesis were detected by keyFluor488 Click-iT EdU detection kit (KeyGene, Nanjing, China). Briefly, cells $\left(2 \times 10^{4}\right.$ cells/well $)$ were inoculated into the 24 -well plates. After $48 \mathrm{~h}$ of treatment, EdU $(50 \mu \mathrm{M})$ was added to the plate for $2 \mathrm{~h}$. After EdU labeling, the cells were fixed in paraformaldehyde (4\%) for $15 \mathrm{~min}$ and then incubated with Triton-X-100 (0.5\%) for $15 \mathrm{~min}$, followed by staining with Click-It reaction mixture for half an hour in a dark place. The nucleic acids were stained using DAPI. Images were photographed under a fluorescence microscope (Olympus, Tokyo, Japan) with a magnification of $\times 200$. All experiments were repeated three times.

\section{Transwell Assay}

Transwell assay was carried out in transwell chambers (Corning Costar, Corning, NY, USA) to assess the migration and invasion of HGC-27 and AGS. In short, cells $\left(2 \times 10^{4}\right.$ cells/well $)$ in serum-free medium $(0.2 \mathrm{~mL})$ were seeded into the top chamber precoated with (invasion assay) or without (migration assay) Matrigel (BD Biosciences, Franklin, NJ, USA). Meanwhile, DMEM with $10 \%$ FBS $(0.6 \mathrm{~mL})$ was added into the bottom chamber (as the chemokine). After incubation for 24 $\mathrm{h}$ at $37^{\circ} \mathrm{C}$ with $5 \% \mathrm{CO}_{2}$, non-migrating and noninvading cells from the top surface of the insert were gently removed with a cotton swab, and the migrating or invading cells were fixed with $4 \%$ paraformaldehyde (Sangon Biotech) for $20 \mathrm{~min}$, and stained with $0.1 \%$ violet solution (Beyotime). The number of migrating or invading cells was calculated using a microscope (Olympus) with a magnification of $\times 100$. All experiments were repeated three times.

\section{Flow Cytometry Analysis}

Cell apoptosis was assessed via an Annexin V-fluorescein isothiocyanate (FITC) and propidium iodide (PI) Apoptosis Detection kit (Sangon Biotech). Briefly, cells $\left(5 \times 10^{5}\right.$ cells/well) were inoculated in 6-well plates and harvested after treatment. After resuspension in binding buffer $(0.4 \mathrm{~mL})$, Annexin V-FITC $(10 \mu \mathrm{L})$ and PI $(5 \mu \mathrm{L})$ were used to stain cells for $0.5 \mathrm{~h}$ in the darkness, followed by detection of apoptotic cells using a flow cytometer (BD Biosciences). All experiments were repeated three times.

\section{Western Blot Assay}

Cells were lysed using RIPA lysis buffer (Beyotime) on ice and centrifuged $\left(20 \mathrm{~min}, 14,000 \mathrm{~g}, 4^{\circ} \mathrm{C}\right)$ to extract total protein. Thereafter, the protein samples were denatured by heating at $100^{\circ} \mathrm{C}$ for $3-5$ min. After measuring protein concentration with BCA protein assay kit (Abcam, Cambridge, UK), extracted protein samples (about $30 \mu \mathrm{g} /$ lane) were separated using sulfate-polyacrylamide gel electrophoresis (SDS-PAGE; Sangon Biotech), and protein was then transferred to nitrocellulose membranes (Invitrogen). After blocking with 5\% skim milk (Beyotime), the membranes were then incubated with the primary antibodies at $4^{\circ} \mathrm{C}$ for $12-16 \mathrm{~h}$ : B-cell lymphoma-2 (Bcl-2; ab194583, 1:500, Abcam), BCL2-associated $X$ protein (Bax; ab77566, 1:1000, Abcam), SOX4 (ab70598, 1:500, Abcam), $\beta$-actin (ab227387, 1:5000, Abcam). Afterwards, these membranes were probed with corresponding secondary antibody (ab205718/ab205719, 1:4000, Abcam). Finally, the combined signals were detected by enhanced chemiluminescence reagent (Solarbio, Beijing, China). Quantification of protein levels was determined using ImageJ software. The protein abundance was normalized by $\beta$-actin. All experiments were repeated three times.

\section{Quantitative Real-Time PCR (qRT-PCR)}

Total RNA was isolated using TRIzol (Invitrogen). Next, the purity and concentration of RNA samples were detected by the measurement of the absorbance at 260 and $280 \mathrm{~nm}$ using NanoDrop 2000 Spectrophotometer (Thermo Scientific, Wilmington, DE, USA). OD260/ OD280 ratio ranged from 18 to 2.1 could be classified as qualified sample. Next, RNA samples $(1 \mu \mathrm{g})$ were reversetranscribed into cDNA using a Primescript RT Reagent (TaKaRa, Kusatsu, Japan) for analysis of circRNA and mRNA, or miRNA1st Strand cDNA Synthesis kit (Vazyme, Nanjing, China) for detection of miRNA. Then qRT-PCR was performed by using BeyoFast ${ }^{\mathrm{TM}}$ SYBR Green qPCR Mix (Beyotime) on CFX96 Touch RealTime PCR Detection System (Bio-Rad). Primer information was listed: circPDSS1 (forward 5'-3': GTGGTG CATGAGATCGCCTA; reverse 5'-3': GGGTTGTG TGATGAAACCTGC); PDSS1 (forward 5'-3': TCTG AAGCTCGGGTTAGCCACT; reverse 5'-3': GCTCTGTCTACATCTCCAGGCA); miR-1324 (forward 5'-3': GCCGAGCCAGACAGAATTCTAT; reverse 5'-3': CAGTGCGTGTCGTGGAGT); SOX4 (forward 5'-3': 
GGCCTCGAGCTGGGAATCGC; reverse 5'-3': GCCC ACTCGGGGTCTTGCAC). $\beta$-Actin (forward 5'-3': GCC GGGACCTGACTGACTAC; reverse $5^{\prime}-3^{\prime}:$ TCTCC TTAATGTCACGCACGAT), U6 (forward 5'-3': CTCG CTTCGGCAGCACATATACT; reverse 5'-3': ACGCT TCACGAATTTGCGTGTC). The RNA levels were assessed with $2-{ }^{\Delta \Delta \mathrm{Ct}}$ method, followed by normalization to $\beta$-actin (for circPDSS1 and SOX4) and U6 (for miR1324). All experiments were repeated three times.

\section{RNase R Treatment}

RNase R treatment was utilized for degrading linear RNA. Briefly. RNA $(2 \mu \mathrm{g})$ was incubated with (RNase $\left.\mathrm{R}^{+}\right)$or without (Mock) RNase R (Epicentre Technologies, Madison, WI, USA) for half an hour at $37^{\circ} \mathrm{C}$. After that, the treated RNAs were used for qRT-PCR to evaluate the expression of circPDSS1 and PDSS1. All experiments were repeated three times.

\section{Dual-Luciferase Reporter Assay}

Circinteractome (https://circinteractome.nia.nih.gov/) or DianaTools-microT_CDS (http://diana.imis.athenainnovation.gr/DianaTools/index.php? $\mathrm{r}=$ microT CDS/ index) was applied to predict the potential binding sequence between miR-1324 and circPDSS1 or SOX4. The fragments of circPDSS1 or SOX4 containing the predicated complementary sequences of miR-1324 were amplified and cloned into pmirGLO Dual-luciferase vector (GenePharma, Shanghai, China) to create circPDSS1 WT/MUT and SOX4 3'UTR WT/MUT reporter plasmids. After that, HGC-27 and AGS $\left(2 \times 10^{4}\right.$ cells/well) were inoculated into the 24-well plates and then each of the above-mentioned plasmids and miR-1324/miR-NC were co-transfected into HGC-27 and AGS cells. After being incubated for $48 \mathrm{~h}$, the luciferase activities were measured by dual-Luciferase Reporter Assay System (Promega, Madison, WI, USA). All experiments were repeated three times.

\section{RNA Pull-Down Assay}

Biotin labeled miR-NC (Bio-NC) and miR-1324 (Bio-miR -1324) were bought from GenePharma (Shanghai, China), and were individually transfected into $\mathrm{GC}$ cells for 48 h. After that, cells were harvested and lysed, followed by incubation with streptavidin-coated magnetic beads (Invitrogen). The RNA complexes combining on the beads were washed and used for detection of the enrichment of
circPDSS1 by qRT-PCR. All experiments were repeated three times.

\section{Tumor Formation Assay in vivo}

$\mathrm{BALB} / \mathrm{c}$ nude mice (female, 6 weeks old, $\mathrm{n}=24$, weighing $20-25$ g, Vital River, Beijing, China) were utilized to establish xenograft model. Briefly, HGC-27 cells or cells transfected with circPDSS1 or Vector were inoculated into nude mice. After 7 days of injection, nude mice were randomly divided into four groups: Mock group, propofol group, propofol + Vector, and propofol $+\operatorname{circPDSS} 1$ ( $\mathrm{n}=6 /$ group). The propofol groups was intraperitoneally injected with $(45 \mathrm{mg} / \mathrm{kg})$ twice a week. Mock group was injected with PBS. Tumor volume was calculated and measured every 7 days. These mice were sacrificed 28 days later, and tumor masses were collected. The animal experiments were approved by the Animal Care and Use Committee of Wuhu Hospital of Traditional Chinese Medicine. Animal studies were performed in compliance with the ARRIVE guidelines and the Basel Declaration. All animals received humane care according to the National Institutes of Health (USA) guidelines.

\section{Immunohistochemistry (IHC) Analysis}

Tissues were fixed in $10 \%$ formalin, embedded in paraffin and cut into thick sections $(5 \mu \mathrm{m})$. Next, these sections were incubated with primary antibody against SOX4 (ab243041, 1:1000, Abcam) for $12 \mathrm{~h}$ at $4^{\circ} \mathrm{C}$. Thereafter, the sections were continuously incubated with secondary antibody (ab205719, 1:2000, Abcam) for $1 \mathrm{~h}$. After that, the slides were then stained by 3,3'Diaminobenzidine (DAB) solution (Sigma-Aldrich) and subsequently counterstained with hematoxylin (SigmaAldrich). Finally, images were obtained using a microscope with a magnification of $\times 200$. All experiments were repeated three times.

\section{Statistical Analysis}

All data from at least three independent experiments were displayed as mean \pm standard deviation (SD). Data analysis was performed by GraphPad Prism (GraphPad Software, San Diego California, USA). Student's $t$-test or a one-way analysis of variance was used for calculating significant differences. Statistical significance was considered when $P<0.05$. 


\section{Results}

\section{Propofol Inhibited Cell Proliferation and} Migration and Invasion and Induced Apoptosis in GC Cell

To investigate the functions of propofol in GC cells, HGC27 and AGS cells were exposed to different doses of propofol. CCK-8 and colony formation assays showed that propofol treatment reduced HGC-27 and AGS cell viability in a dose-dependent manner, and also decreased colony formation ability and EdU-positive cells (Figure 1A-D), suggesting that propofol inhibited GC cell proliferation. Meanwhile, transwell assay indicated that propofol treatment suppressed HGC-27 and AGS cell migration and invasion in a dose-dependent manner (Figure 1E and F). Moreover, propofol dose-dependently promoted HGC-27 and AGS cell apoptosis (Figure 1G). Next, Western blot assay was performed to measure the expression levels of apoptosis-related proteins (Bcl-2, an anti-apoptotic molecule; Bax, a pro-apoptotic molecule). As presented in Figure 1H, propofol increased the protein expression of Bax and decreased the protein expression of Bcl-2. These data collectively indicated that propofol might play an anticancer role in GC.

\section{Propofol Reduced the Expression of circPDSSI in GC Cells}

Next, we explored the effect of propofol on the expression of circPDSS1. As displayed in Figure 2A and B, the expression of circPDSS1 was decreased by treatment with propofol in HGC-27 and AGS cells. The data from
A

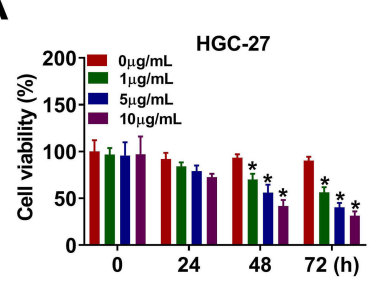

D

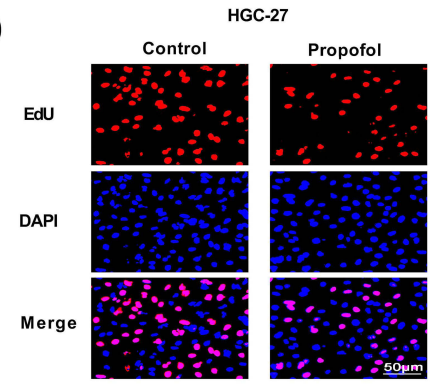

B

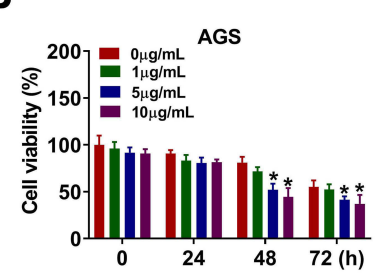

C
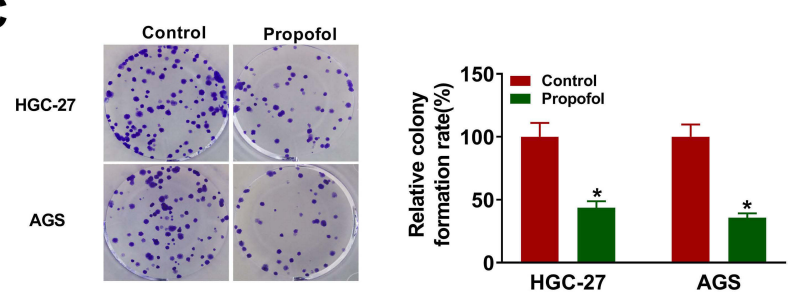

E

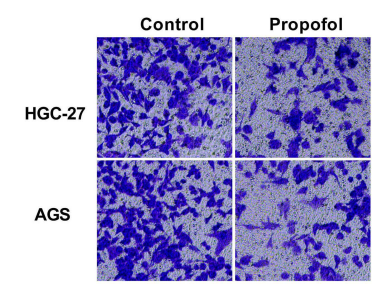

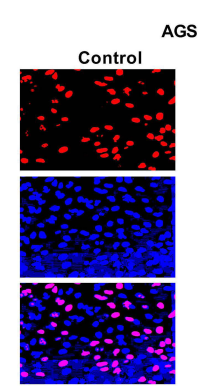

AGS

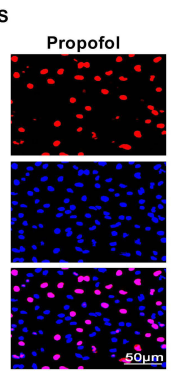

$\mathbf{F}$
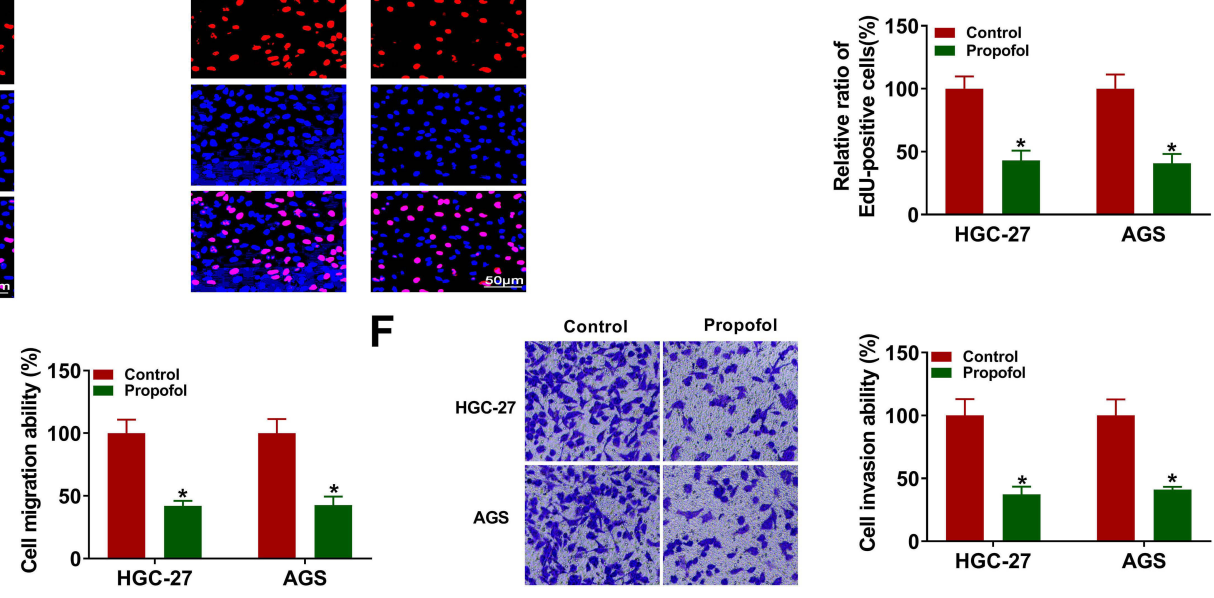

G
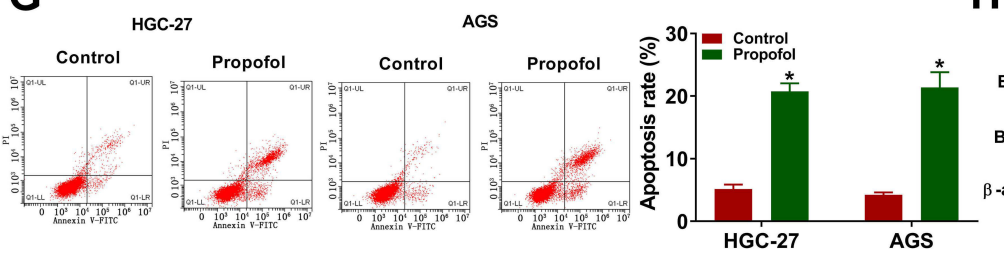

H
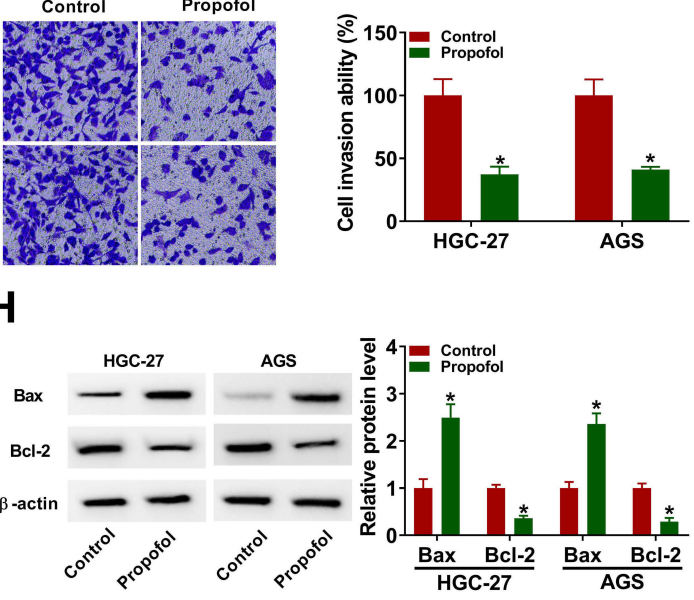

Figure I Propofol exerted anticancer role in GC cells. (A and B) Cell viability was detected using CCK-8 assay in HGC-27 and AGS cells treated with different concentrations of propofol. (C-G) HGC-27 and AGS cells were treated with or without propofol $(10 \mu \mathrm{g} / \mathrm{mL})$. (C) Colony formation assay was utilized to measure colony formation ability. (D) DNA synthesis was determined by EdU assay $(\times 200)$. (E and $\mathbf{F})$ Transwell assay was utilized to evaluate cell migration and invasion assay $(\times 100)$. (G) Flow cytometry analysis was used to detect cell apoptosis rate. $(\mathbf{H})$ The protein levels of Bax and Bcl-2 were determined by Western blot assay. $* P<0.05$. 
A

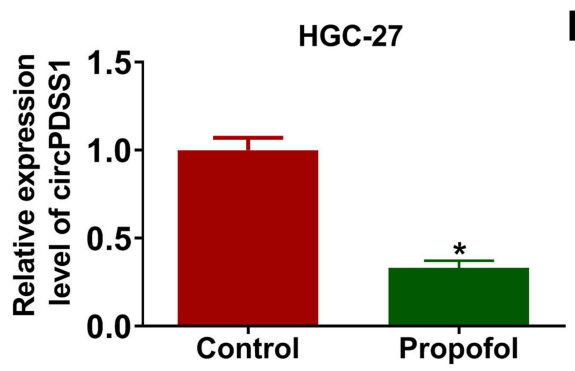

C

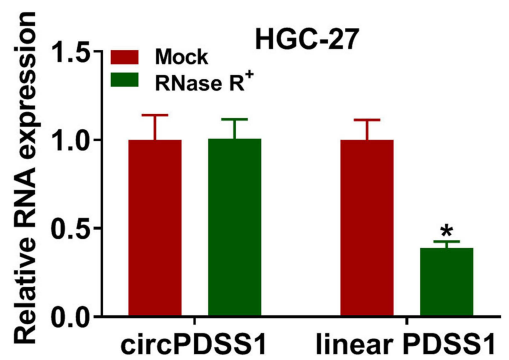

B

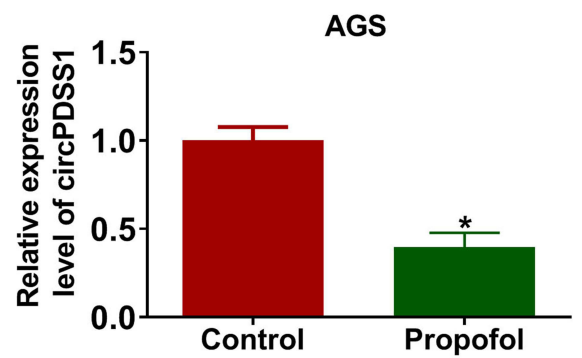

D

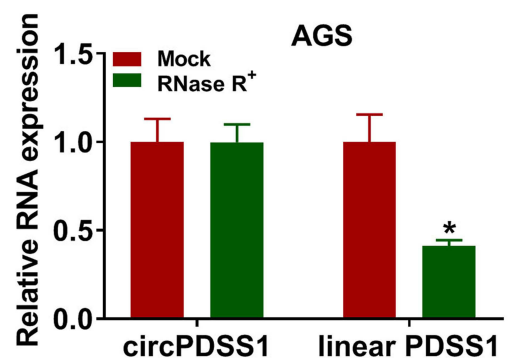

Figure 2 Propofol treatment decreased the expression of circPDSSI in GC cells. (A and B) The expression of circPDSSI was measured by qRT-PCR in HGC-27 and AGS cells treated with or without propofol. (C and D) The levels of circPDSSI and linear PDSSI were examined after treatment of RNase R by qRT-PCR in HGC-27 and AGS cells. $* p<0.05$

qRT-PCR showed that circPDSS1 was resistant to RNase $\mathrm{R}$ digestion (Figure $2 \mathrm{C}$ and $\mathrm{D}$ ), indicating the cyclic structure of circPDSS1. Our results revealed that circPDSS1 expression was decreased in GC cells treated with propofol and circPDSS1 had a closed-loop structure.

\section{Propofol Exerted the Anticancer Role by Downregulating circPDSSI in GC Cells}

Based on the regulatory relationship between circPDSS1 and propofol, we further explored whether circPDSS1 was involved in propofol-mediated inhibition on GC cell progression. The data of qRT-PCR showed that the inhibitory effect of propofol on the expression of circPDSS1 was reversed by circPDSS1 upregulation in HGC-27 and AGS cells (Figure 3A and B). Moreover, circPDSS1 overexpression abated the suppressive effects of propofol on cell viability, colony-forming ability, EdU-positive cells, migration, and invasion in HGC-27 and AGS cells (Figure 3C-H). Furthermore, upregulation of circPDSS1 weakened propofol-induced apoptosis in HGC-27 and AGS cells (Figure 3I). Also, transfection of circPDSS1 reversed the promotion of Bax protein expression and reduction of $\mathrm{Bcl}-2$ protein expression caused by propofol (Figure 3J and $\mathrm{K}$ ). However, transfection of circPDSS1 MUT had little effect on the expression of circPDSS1 (Supplementary Figure 1A and B). Meanwhile, overexpression of circPDSS1 MUT did not affect GC cell proliferation, migration and invasion, (Supplementary Figure 1C-H). Collectively, propofol inhibited GC cell tumorigenesis by regulating circPDSS1.

\section{circPDSSI Acted as a Sponge of miR-I324 in GC Cells}

To elucidate the underlying mechanism of circPDSS1 in GC, the potential miRNA targets of circPDSS1 were predicted using circinteractome. We found that there were binding sites between circPDSS1 and miR-1324 (Figure 4A), indicating that miR-1324 might be a target of circPDSS1. Overexpression or inhibition efficiency of miR-1324 was determined by qRTPCR. The data showed that transfection of miR-1324 increased the expression of miR-1324 and transfection of anti-miR-1324 decreased the expression of miR-1324 in HGC-27 and AGS cells (Figure 4B). To confirm the interaction between circPDSS1 and miR-1324, dual-luciferase reporter assay and RNA pull-down assay were performed. We found that miR1324 overexpression reduced the luciferase activity of circPDSS1 WT but not the activity of circPDSS1 MUT in HGC-27 and AGS cells (Figure 4C and D). RNA pull-down assay exhibited that transfection of Bio-miR-1324 led to an increased in the enrichment of circPDSS1 in HGC-27 and AGS cells (Figure 4E). Afterwards, HGC-27 and AGS cells were treated with propofol, and qRT-PCR analysis suggested that propofol treatment increased the expression of miR-1324 
A

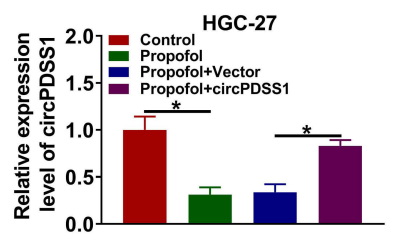

E

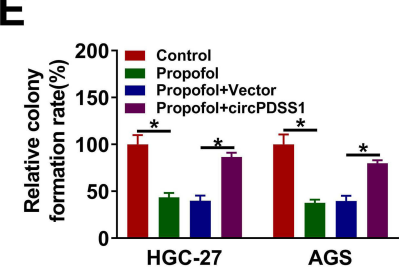

B

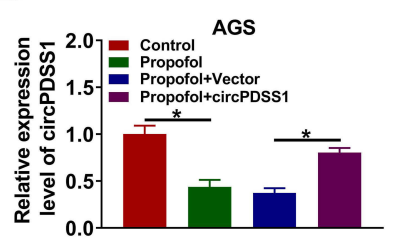

F

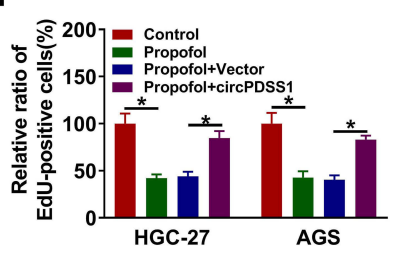

C

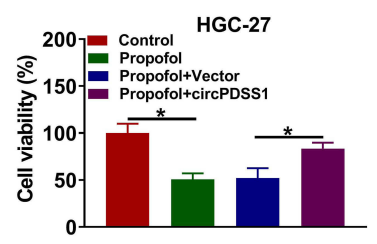

G

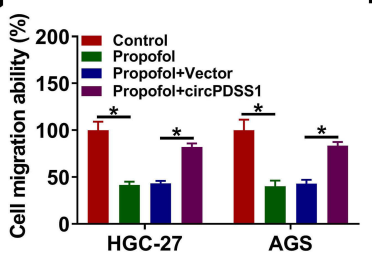

D

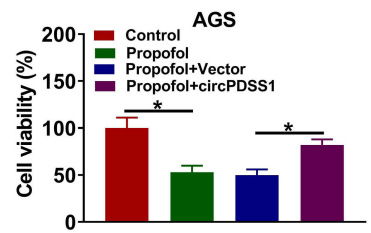

H

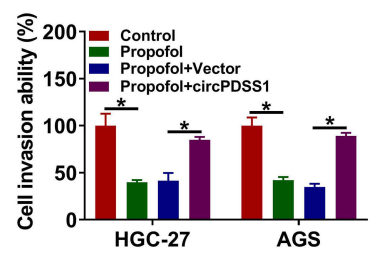

I

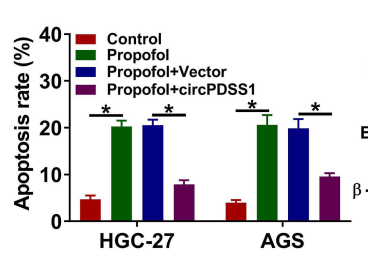

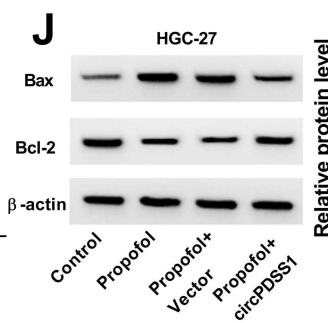
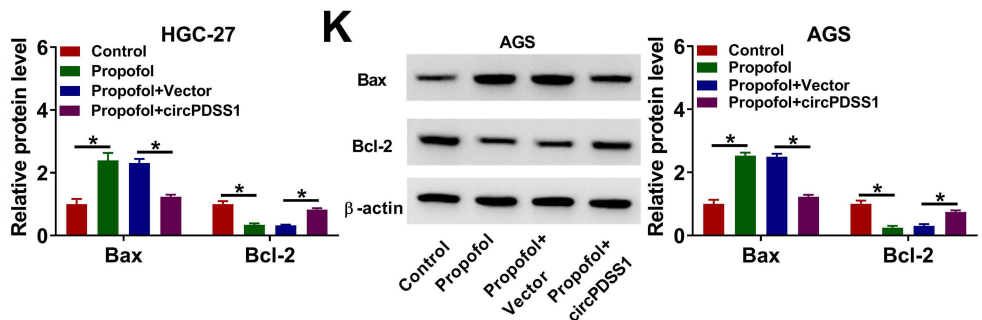

Figure 3 Propofol inhibited GC cell progression by regulating circPDSSI. HGC-27 and AGS cells were divided into four groups: Control, Propofol, Propofol + Vector, and Propofol + circPDSSI. (A and B) The expression of circPDSSI was determined using qRT-PCR. (C and D) Cell viability was assessed by CCK-8 assay. (E) Cell colonyforming ability was evaluated by colony formation assay. (F) DNA synthesis was examined using EdU assay ( $\mathbf{G}$ and $\mathbf{H})$ Cell migration and invasion were determined by transwell assay. (I) Flow cytometry analysis was used for detecting cell apoptosis. ( $\mathbf{J}$ and $\mathbf{K}$ ) The protein levels of Bax and $\mathrm{Bcl}-2$ were analyzed by $W$ estern blot assay. $* P<0.05$.

(Figure 4F and G). Moreover, we observed that transfection of si-circPDSS1 inhibited the expression of circPDSS1 (Figure 4H), implying that circPDSS1 was successfully knocked down. Next, we explored the impact of circPDSS1 on miR-1324 expression. As shown in Figure 4I, the expression of miR-1324 was increased by circPDSS1 downregulation. These results revealed that miR-1324 was a target of circPDSS1.

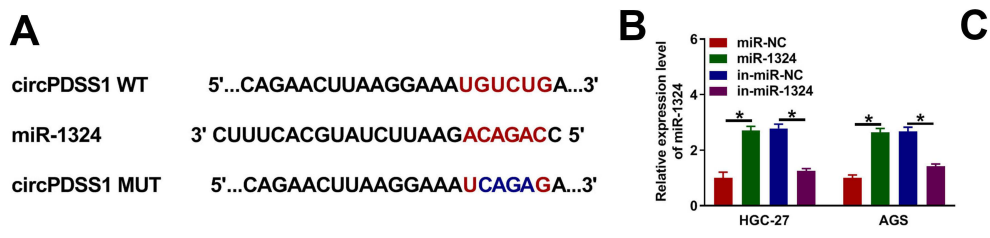

E

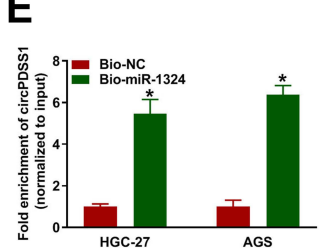

$\mathbf{F}$

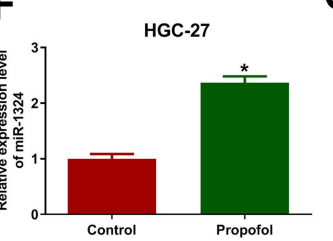

G

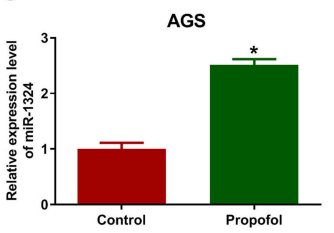

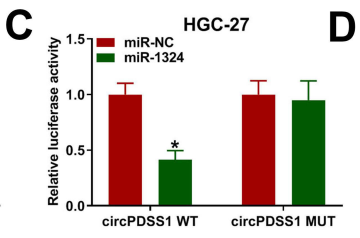

H

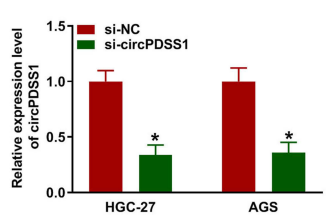

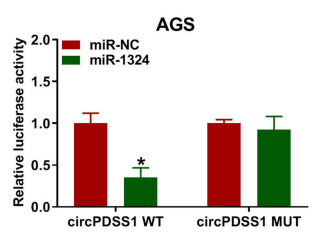

I

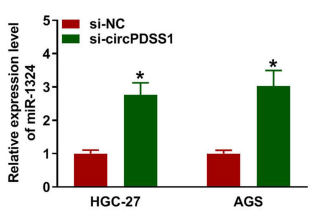

Figure 4 MiR-I 324 was a target of circPDSSI in GC cells. (A) The binding sequence between miR-I 324 and circPDSSI was presented. (B) The expression of miR-I324 was measured by qRT-PCR in HGC-27 and AGS cells were transfected with miR-NC, miR-1324, in-miR-NC, or in-miR-I324. (C and D) Dual-luciferase reporter assay was conducted to measure the luciferase activity in HGC-27 and AGS cells co-transfected with circPDSSI WT or circPDSSI MUT and miR-NC or miR-I324. (E) The enrichment of circPDSSI was examine by RNA pull-down assay in HGC-27 and AGS cells transfected with Bio-NC or Bio-miR-I324. (F and G) The expression level of miR-I324 was detected by qRT-PCR in HGC-27 and AGS cells treated with or without propofol. (H and I) The expression levels of circPDSSI and miR-I324 were detected by qRT-PCR in HGC-27 and AGS cells transfected with si-NC or si-circPDSSI. $* P<0.05$. 


\section{circPDSSI Promoted Cell Proliferation}

and Migration and Invasion and Inhibited

Apoptosis by Downregulating miR-I324

\section{in Propofol-Treated GC Cells}

Next, we explored the whether the effects of circPDSS1 were mediated by miR-1324 in propofoltreated GC cells. The expression of miR-1324 was reduced by overexpression of circPDSS1, which was rescued by upregulating miR-1324 (Figure 5A and B). Moreover, circPDSS1 overexpression promoted cell viability, colony-forming ability, EdU-positive cells, migration and invasion, while these effects were abated by elevating miR-1324 (Figure 5C-H). In addition, circPDSS1 upregulation reduced cell apoptosis and Bax protein expression and increased the expression of Bcl-2, which could be revered by miR-1324 overexpression (Figure 5I-K). Taken together, these data suggested that circPDSS1 exerted its role by sponging miR-1324 in propofol-treated GC cells.

\section{SOX4 Was a Target of miR-I324 in GC Cells}

To elucidate which genes are regulated by the circPDSS1/ miR-1324 axis in GC, DianaTools-microT_CDS was used to predict the targets of miR-1324. As presented in Figure $6 \mathrm{~A}$, SOX4 might be a target of miR-1324. The results of dual-luciferase reporter assay showed that miR1324 upregulation considerably decreased the luciferase activity of SOX4 3'UTR WT compared with the miR-NC group, while miR-1324 overexpression did not have any impact on the luciferase activity of SOX4 3'UTR MUT (Figure 6B and $\mathrm{C}$ ). Moreover, propofol treatment reduced the protein expression of SOX4 in HGC-27 and AGS cells (Figure $6 \mathrm{D}$ and $\mathrm{E}$ ). In addition, the protein expression of SOX4 was inhibited by miR-1324 overexpression in HGC-27 and AGS cells (Figure 6F). Moreover, we found that circPDSS1 expression and SOX4 mRNA expression were higher in GC tissues than that in normal tissues, while miR-1324 expression was lower in GC tissues (Supplementary Figure 2A-C). In addition, we observed
A

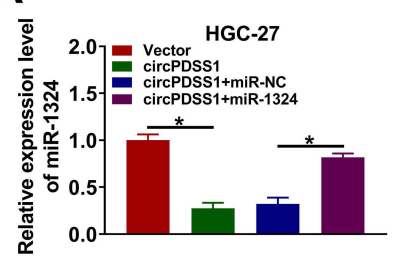

$E$

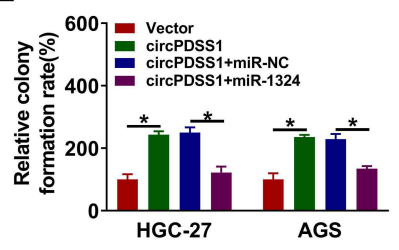

B

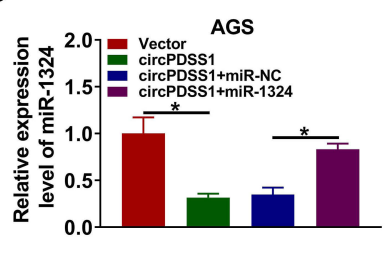

$\mathbf{F}$

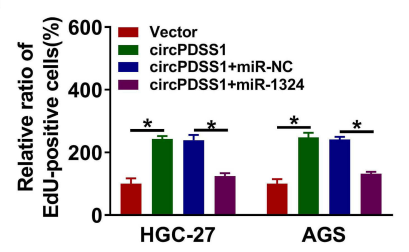

C

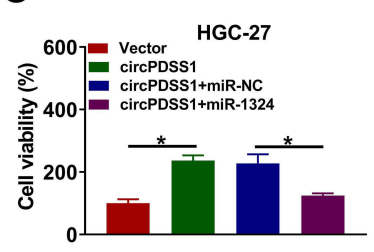

G

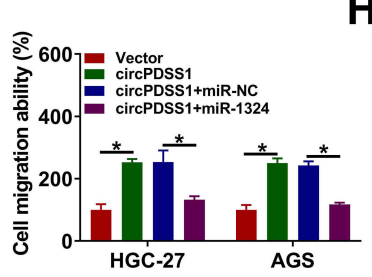

D

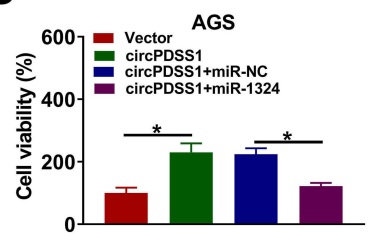

H

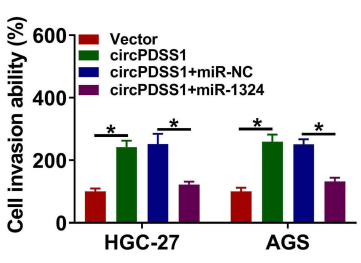

I

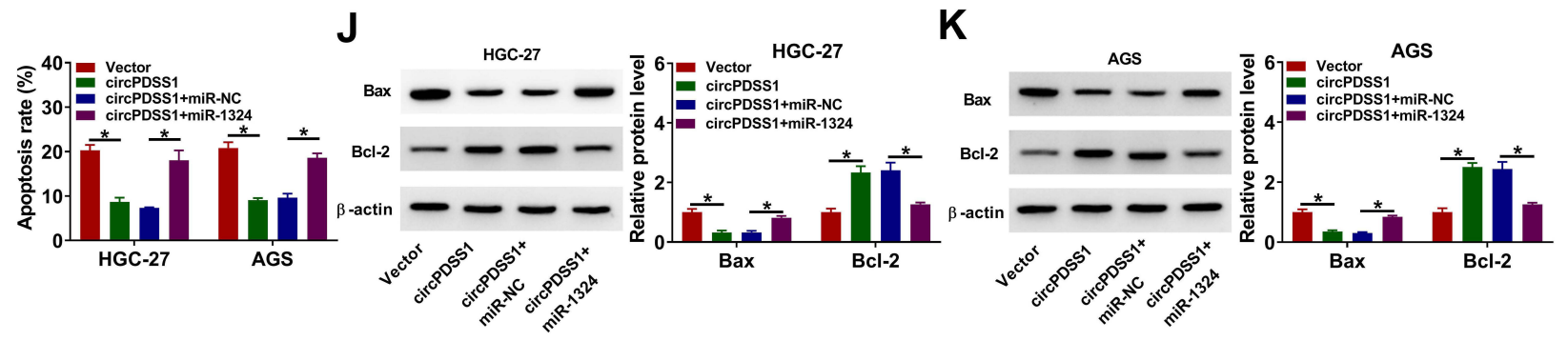

Figure 5 Overexpression of circPDSSI promoted GC cell progression by downregulating miR-I 324 in propofol-treated GC cells. HGC-27 and AGS cells were transfected with Vector, circPDSSI, circPDSSI + miR-NC, or circPDSSI + miR-I324 and then treated with propofol. (A and B) The expression level of miR-I324 was tested by qRTPCR. (C-F) CCK-8, colony formation, and EdU assays were used to assess cell proliferation ability. ( $\mathbf{G}$ and $\mathbf{H}$ ) Transwell assay was used for measuring cell migration and invasion abilities. (I) Cell apoptosis was measured using flow cytometry analysis. ( $\mathbf{J}$ and $\mathbf{K}$ ) Western blot assay was performed to detect the protein levels of Bax and Bcl-2. $* P<0.05$. 


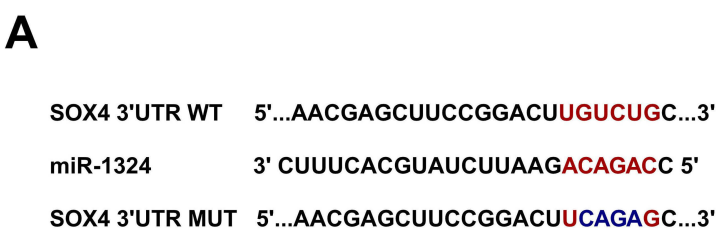

D

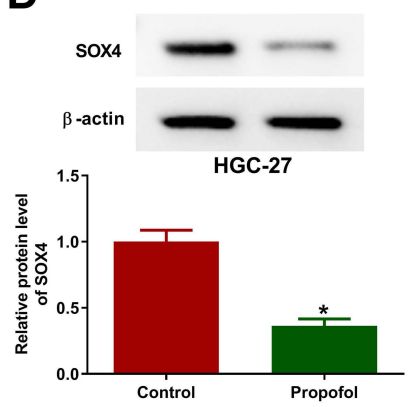

E

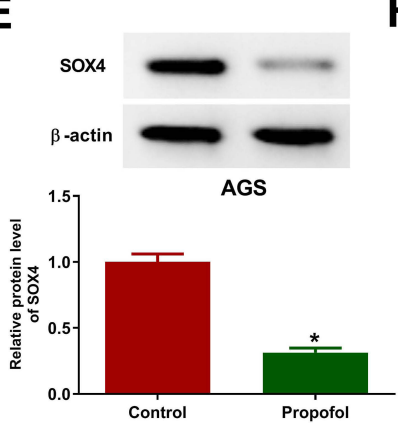

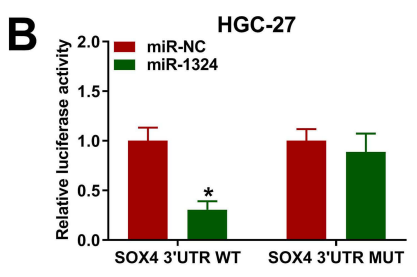

$\mathbf{F}$

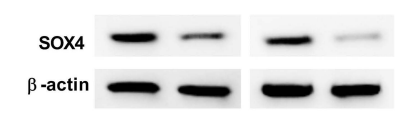

G

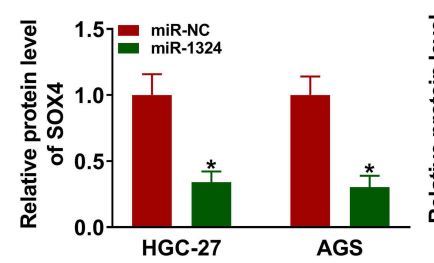

Figure 6 SOX4 was directly targeted by miR-I324 in GC cells. (A) The complementary binding sequence of miR-I324 and SOX4 3'UTR was shown. (B and C) Luciferase activity was measured in HGC-27 and AGS cells co-transfected with SOX4 3'UTR WT and SOX4 3'UTR MUT and miR-NC or miR-I324. (D and E) The protein expression of SOX4 was measured by Western blot assay in HGC-27 and AGS cells treated with or without propofol. (F) Western blot assay was conducted to detect the protein expression of SOX4 in HGC-27 and AGS cells transfected with miR-NC or miR-1324. (G) The protein level of SOX4 was determined by Western blot assay in HGC-27 and AGS cells transfected with si-NC, si-circPDSSI, si-circPDSSI + in-miR-NC, or si-circPDSSI + in-miR-I324. $* P<0.05$.

that miR-1324 expression was negatively correlated with circPDSS1 expression and SOX4 mRNA expression in GC tissues, and circPDSS1 expression was positively correlated with SOX4 mRNA expression in GC tissues (Supplementary Figure 2D-F). Subsequently, we determined whether circPDSS1 was able to act as a miR-1324 sponge to regulate SOX4. Results revealed that circPDSS1 silencing suppressed the protein expression of SOX4 in HGC-27 and AGS cells, which was restored by inhibition of miR-1324 (Figure 6G), indicating that circPDSS1 positively regulated SOX4 expression by sponging miR-1324.

\section{Overexpression of miR-I324 Suppressed Cell Proliferation and Migration and Invasion and Facilitated Apoptosis by Targeting SOX4 in Propofol-Treated GC Cells}

To determine whether the role of miR-1324 in GC was regulated by SOX4, HGC-27 and AGS cells were transfected with miR-NC, miR-1324, miR-1324 + pcDNA, or miR-1324 + SOX4 prior to propofol exposure. The protein expression of SOX4 was reduced by transfection of miR1324, which was restored by co-transfection of SOX4 (Figure 7A and B). Overexpression of miR-1324 inhibited cell viability, colony formation ability, EdU-positive cells, migration, and invasion, while SOX4 enhancement reversed these effects (Figure 7C-H). Meanwhile, enforced expression of miR-1324 increased cell apoptosis and the protein expression of Bax as well as reduced the protein expression of Bcl-2 expression, which could be counteracted by upregulating SOX4 (Figure 7I-K). These results indicated that miR-1324 exerted its role by targeting SOX4 in propofol-treated GC cells.

\section{circPDSSI Weakened the Effect of Propofol on Inhibition of Tumor Growth in vivo}

To determine the roles of propofol and circPDSS1 in tumor growth in vivo, we established mice xenograft model. As presented in Figure 8A and B, propofol treatment decreased tumor volume and weight, while overexpression of circPDSS1 could neutralize this inhibitory effect. Moreover, propofol treatment reduced the expression level of circPDSS1 and the protein level of SOX4 as well as enhanced the expression level of miR-1324 in tumor tissues, while these effects were reversed by overexpression of circPDSS1 (Figure 8C-E). IHC analysis showed that propofol also reduced the expression of SOX4, which was restored by upregulating circPDSS1 (Figure 8F). Collectively, propofol suppressed tumor growth in vivo by downregulating circPDSS1.

\section{Discussion}

An increasing number of reports have shown that propofol possesses anti-anxiety, neuroprotective, anti-oxidation and 

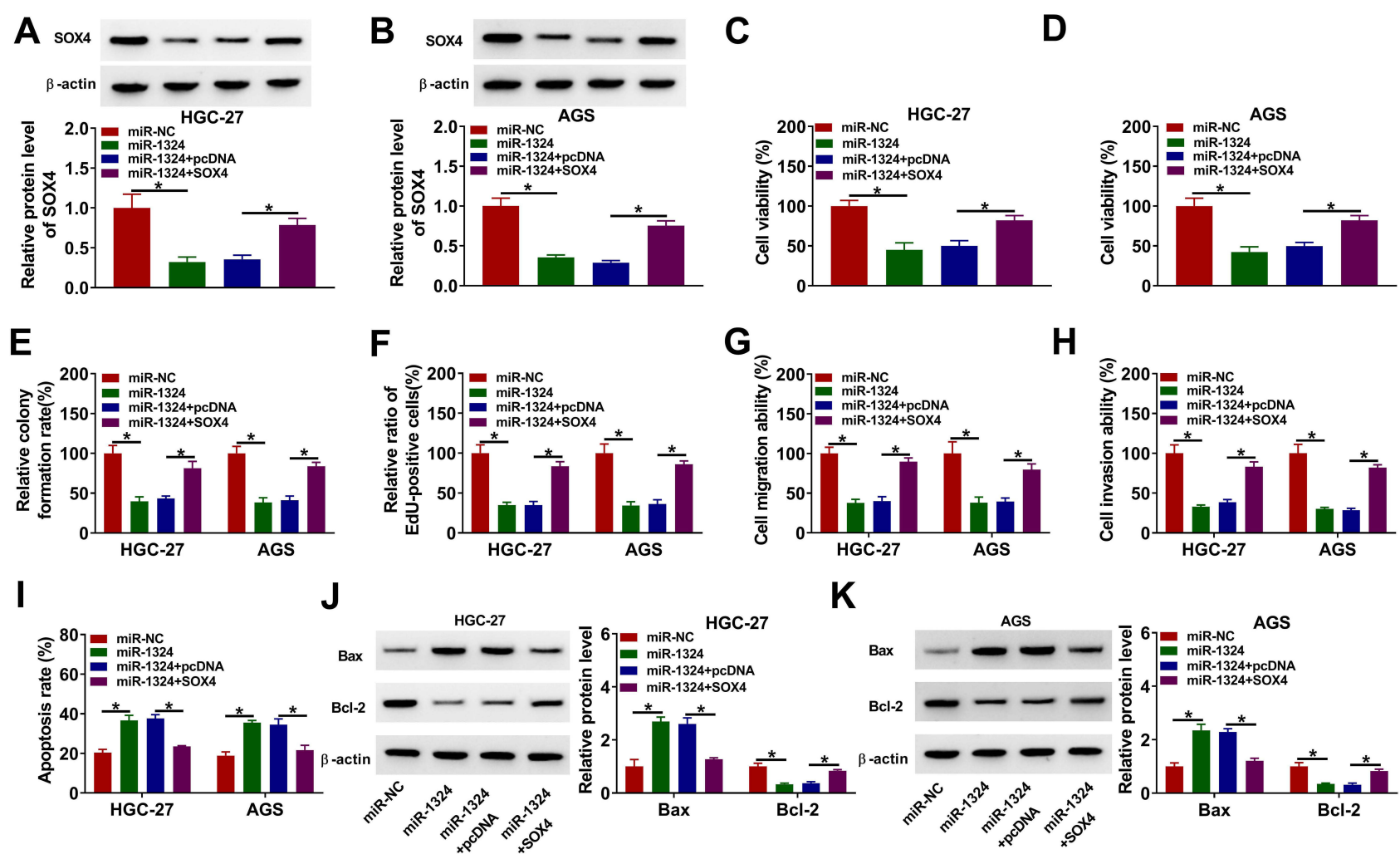

Figure 7 MiR-1324 suppressed GC cell progression by targeting SOX4 in propofol-treated GC cells. HGC-27 and AGS cells were transfected with miR-NC, miR-I324, miR1324 + pcDNA, or miR-1324 + SOX4, followed by treatment with propofol. (A and B) The protein expression of SOX4 was analyzed by Western blot assay. (C-F) Cell proliferation ability was assessed by CCK-8 assay, colony formation assay, and EdU assay. ( $\mathbf{G}$ and $\mathbf{H}$ ) Cell migration and invasion were measured by transwell assay. (I) Flow cytometry analysis was utilized to determine cell apoptosis. ( $\mathbf{J}$ and $\mathbf{K})$ The protein levels of Bax and Bcl-2 were determined by Western blot assay. ${ }^{* P}<0.05$.

antitumor roles in some diseases. ${ }^{25-27}$ In this research, we identified the anticancer role of propofol in the biological behaviors of GC cells, and we uncovered that propofol suppressed GC cell proliferation, migration and invasion and facilitated apoptosis and also inhibited tumor growth in vivo, which was in agreement with previous results. For instance, Zhu et al indicated that propofol repressed proliferation and metastasis of GC cells via modulation of miR-140-5p. ${ }^{28}$ A report by Yang et al also declared that propofol suppressed the growth and survival of GC cells in vitro by upregulating ING3. ${ }^{29}$ However, how propofol exerts anticancer effect in GC remains largely unknown.

Next, we explored the molecular mechanism related to propofol in GC. Some studies have shown that propofol exert antitumor functions in some cancers through regulating circRNAs. ${ }^{30,31}$ Therefore, we explored whether propofol suppressed the progression of GC via regulating circRNA. Some studies revealed that circPDSS1 served as a tumor promotor in some cancers. Fang et al pointed out that circPDSS1 was upregulated in colorectal cancer tissues and circPDSS1 promoted migratory ability and angiogenesis in colorectal cancer cells via activating
Wnt/ $\beta$-catenin signaling. ${ }^{32}$ In addition, Yu et al declared that circPDSS1 promoted the development of bladder cancer via downregulation of miR-16. ${ }^{33}$ More importantly, Ouyang et al revealed that circPDSS1 was upregulated in GC tissue samples and cell lines, and knockdown of circPDSS1 repressed GC cell proliferation and accelerated apoptosis via sponging miR-186-5p and regulating NEK2 ${ }^{16}$ Thus, we explored the interaction between propofol and circPDSS1 in GC. In the present study, propofol treatment decreased circPDSS1 expression in GC cells, implying that circPDSS1 might participate in the functions of propofol in GC. More importantly, circPDSS1 upregulation abolished the inhibitory effect of propofol on GC cell progression, suggesting that propofol exerted antitumor function by downregulating circPDSS1.

CircRNAs can regulate the expression of target genes by serving as mRNA sponges. ${ }^{34}$ To explore whether circPDSS1 exerted its role in $\mathrm{GC}$ via sponging miRNAs, bioinformatics analysis (circinteractome) was used. We identified circPDSS1 as a sponge of miR-1324. MiR-1324 is a newly discovered miRNA in recent years. MiR-1324 has been reported to act as a tumor inhibitor in some cancers. ${ }^{35,36}$ 
A

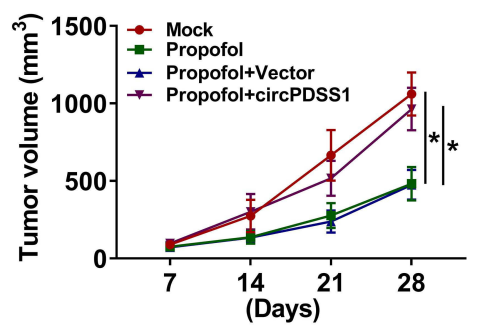

D

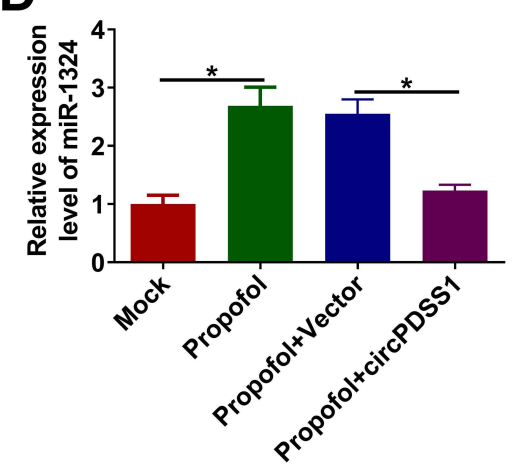

B

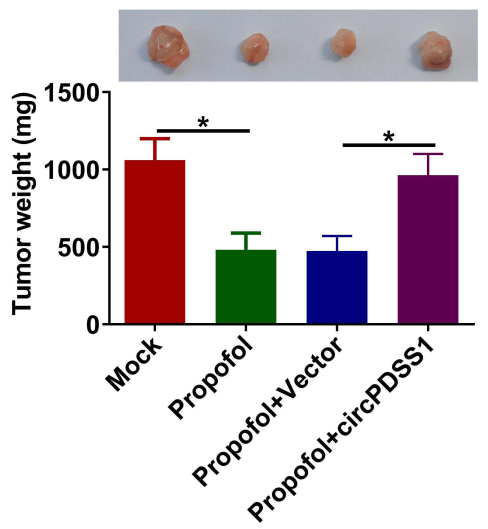

E

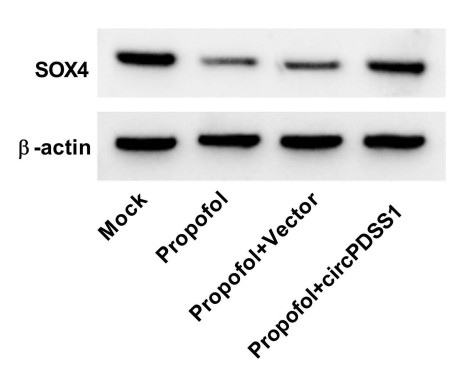

C
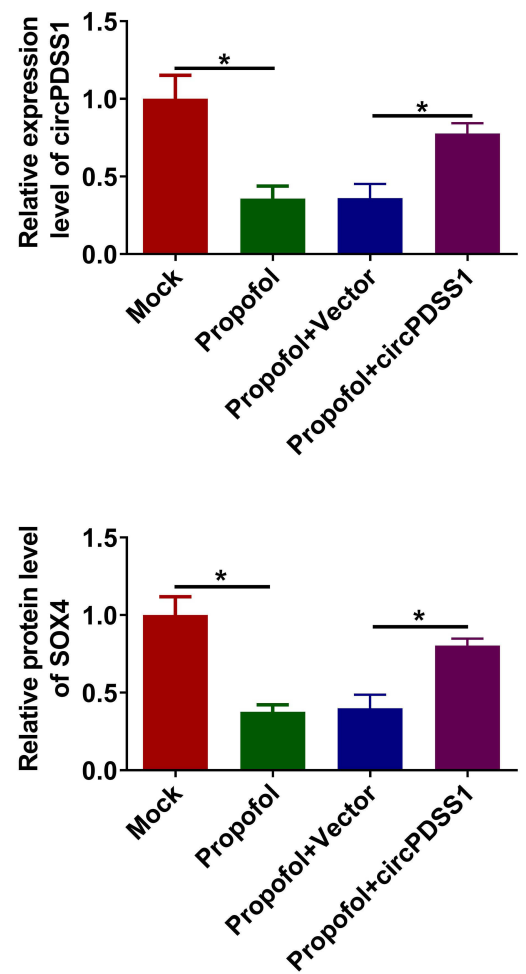

F

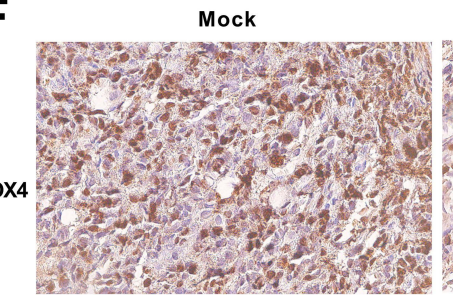

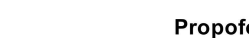

Propofol

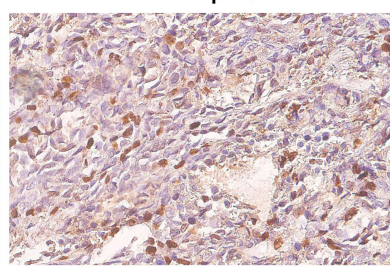

Propofol+Vector

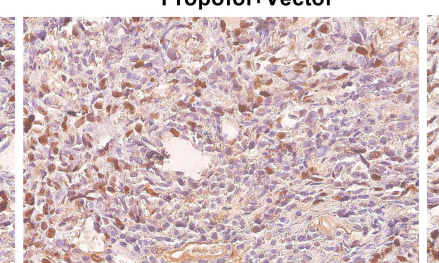

Propofol+circPDSS1

Figure 8 Propofol inhibited tumor growth in vivo by downregulating circPDSSI. HGC-27 cells or HGC-27 cells transfected with circPDSSI (or Vector) were subcutaneously inoculated into the nude mice. After injection for 7 days, the mice were injected with propofol or PBS twice a week. (A) Tumor volume of nude mice was recorded every 7 days. (B) Tumor weight was measured after injection for 28 days. (C and D) The expression levels of circPDSSI and miR-I324 were examined using qRT-PCR in resected tumor tissues. (E) The protein level of SOX4 was measured by Western blot in resected tumor tissues. (F) The expression of SOX4 in tissues was examined by IHC analysis $(\times 200)$. $* P<0.05$.

Additionally, Zhang et al reported that miR-1324 was downregulated in GC, and inhibition of miR-1324 enhanced the proliferative capacity and invasion of GC cells by regulating MECP $2 .{ }^{20}$ In this research, miR-1324 level was elevated by propofol treatment. Rescue experiments revealed that restoration of miR-1324 abated the impact of circPDSS1 upregulation on decrease of cell proliferation, migration and invasion and increase of apoptosis in propofol-treated GC cells, indicating that propofol inhibited GC cell progression through regulating the circPDSS1/miR-1324 axis. To analyze how miR-1324 influenced the function of circPDSS1 in propofol-treated GC cells, possible targets of miR-1324 were predicted using online tool. We demonstrated that miR1324 directly targeted SOX4.
Recent studies indicated that SOX4 might contribute to the tumor progression by regulating multiple signaling pathways, such as the PI3K/Akt, Wnt and p53 pathway. ${ }^{37-39}$ Moreover, SOX4 has been reported to play a carcinogenic role in GC. For instance, Zhang et al illustrated that SOX4 interference inhibited the malignant behaviors of GC cells. ${ }^{40}$ Pang et al showed that miR-138 inhibited GC progression by downregulating SOX4 in GC. ${ }^{41}$ Here, we found that SOX4 expression was downregulated by propofol treatment. Additionally, we observed that miR-1324 upregulation inhibited GC cell progression by suppressing SOX4 expression in propofolstimulated GC cells. In vivo experiments showed that circPDSS1 overexpression weakened the inhibitory effect 
of propofol on tumor growth by downregulating miR-1324 and upregulating SOX4.

In conclusion, these results showed propofol inhibited GC progression by regulating circPDSS1/miR-1324/SOX4 axis. These findings might provide a theoretical basis for propofol adjuvant treatment of GC.

\section{Funding}

There is no funding to report.

\section{Disclosure}

The authors declare that they have no conflict of interest.

\section{References}

1. Sung H, Ferlay J, Siegel RL, et al. Global cancer statistics 2020: GLOBOCAN estimates of incidence and mortality worldwide for 36 cancers in 185 countries. CA Cancer J Clin. 2021;71(3):209-249. doi: $10.3322 /$ caac. 21660

2. Allemani C, Weir HK, Carreira H, et al. Global surveillance of cancer survival 1995-2009: analysis of individual data for 25,676,887 patients from 279 population-based registries in 67 countries (CONCORD-2). Lancet. 2015;385(9972):977-1010. doi:10.1016/ S0140-6736(14)62038-9

3. Chidambaran V, Costandi A, D'Mello A. Propofol: a review of its role in pediatric anesthesia and sedation. CNS Drugs. 2015;29 (7):543-563.

4. Vasileiou I, Xanthos T, Koudouna E, et al. Propofol: a review of its non-anaesthetic effects. Eur $J$ Pharmacol. 2009;605(1-3):1-8. doi:10.1016/j.ejphar.2009.01.007

5. Qi Z, Yuan L, Sun N. Propofol exhibits a tumor-suppressive effect and regulates cell viability, migration and invasion in bladder carcinoma by targeting the microRNA-10b/HOXD10 signaling pathway. Oncol Lett. 2019;18(6):6228-6236.

6. Yang N, Liang Y, Yang P, Yang T, Jiang L. Propofol inhibits lung cancer cell viability and induces cell apoptosis by upregulating microRNA-486 expression. Braz J Med Biol Res. 2017;50(1):e5794. doi:10.1590/1414-431x20165794

7. Yu X, Gao Y, Zhang F. Propofol inhibits pancreatic cancer proliferation and metastasis by up-regulating miR-328 and down-regulating ADAM8. Basic Clin Pharmacol Toxicol. 2019;125(3):271-278. doi:10.1111/bcpt.13224

8. Li Y, Dong W, Yang H, Xiao G. Propofol suppresses proliferation and metastasis of colorectal cancer cells by regulating miR-124-3p.1/AKT3. Biotechnol Lett. 2020;42(3):493-504. doi:10.1007/s10529-019-02787-y

9. Zhang W, Wang Y, Zhu Z, Zheng Y, Song B. Propofol inhibits proliferation, migration and invasion of gastric cancer cells by up-regulating microRNA-195. Int J Biol Macromol. 2018;120(Pt A):975-984. doi:10.1016/j.ijbiomac.2018.08.173

10. Memczak S, Jens M, Elefsinioti A, et al. Circular RNAs are a large class of animal RNAs with regulatory potency. Nature. 2013;495 (7441):333-338. doi:10.1038/nature11928

11. Shang Q, Yang Z, Jia R, Ge S. The novel roles of circRNAs in human cancer. Mol Cancer. 2019;18(1):6. doi:10.1186/s12943-018-0934-6

12. Zhang Z, Yang T, Xiao J. Circular RNAs: promising biomarkers for human diseases. EBioMedicine. 2018;34:267-274. doi:10.1016/j. ebiom.2018.07.036

13. Sun H, Xi P, Sun Z, et al. Circ-SFMBT2 promotes the proliferation of gastric cancer cells through sponging miR-182-5p to enhance CREB1 expression. Cancer Manag Res. 2018;10:5725-5734. doi:10.2147/ CMAR.S172592
14. Wang Q, Wang T, Hu Y, et al. Circ-EIF4G3 promotes the development of gastric cancer by sponging miR-335. Pathol Res Pract. 2019;215(9):152507. doi:10.1016/j.prp.2019.152507

15. Lu J, Wang YH, Huang XY, et al. circ-CEP85L suppresses the proliferation and invasion of gastric cancer by regulating NFKBIA expression via miR-942-5p. J Cell Physiol. 2020;235(9):6287-6299. doi: $10.1002 /$ jcp.29556

16. Ouyang Y, Li Y, Huang Y, et al. CircRNA circPDSS1 promotes the gastric cancer progression by sponging miR-186-5p and modulating NEK2. J Cell Physiol. 2019;234(7):10458-10469. doi:10.1002/ jcp. 27714

17. Bach DH, Lee SK, Sood AK. Circular RNAs in cancer. Mol Ther Nucleic Acids. 2019;16:118-129. doi:10.1016/j.omtn.2019.02.005

18. Panda AC. Circular RNAs Act as miRNA sponges. Adv Exp Med Biol. 2018;1087:67-79.

19. Gattolliat CH, Uguen A, Pesson M, et al. MicroRNA and targeted mRNA expression profiling analysis in human colorectal adenomas and adenocarcinomas. Eur $J$ Cancer. 2015;51(3):409-420. doi:10.1016/j.ejca.2014.12.007

20. Zhang XY, Xu YY, Chen WY. MicroRNA-1324 inhibits cell proliferative ability and invasiveness by targeting MECP2 in gastric cancer. Eur Rev Med Pharmacol Sci. 2020;24(9):4766-4774.

21. Schepers GE, Teasdale RD, Koopman P. Twenty pairs of SOX: extent, homology, and nomenclature of the mouse and human SOX transcription factor gene families. Dev Cell. 2002;3(2):167-170. doi:10.1016/S1534-5807(02)00223-X

22. Liu P, Ramachandran S, Ali Seyed M, et al. Sex-determining region $Y$ box 4 is a transforming oncogene in human prostate cancer cells. Cancer Res. 2006;66(8):4011-4019. doi:10.1158/0008-5472.CAN-05-3055

23. Wang L, Zhang J, Yang X, et al. SOX4 is associated with poor prognosis in prostate cancer and promotes epithelial-mesenchymal transition in vitro. Prostate Cancer Prostatic Dis. 2013;16 (4):301-307. doi:10.1038/pcan.2013.25

24. Wang CY, Hua L, Sun J, et al. MiR-211 inhibits cell proliferation and invasion of gastric cancer by down-regulating SOX4. Int J Clin Exp Pathol. 2015;8(11):14013-14020.

25. Ulbrich F, Eisert L, Buerkle H, Goebel U, Schallner N. Propofol, but not ketamine or midazolam, exerts neuroprotection after ischaemic injury by inhibition of Toll-like receptor 4 and nuclear factor kappa-light-chain-enhancer of activated B-cell signalling: a combined in vitro and animal study. Eur J Anaesthesiol. 2016;33 (9):670-680. doi:10.1097/EJA.0000000000000449

26. Tian Y, Guo S, Guo Y, Jian L. Anesthetic propofol attenuates apoptosis, a $\beta$ accumulation, and inflammation induced by sevoflurane through NF-kb pathway in human neuroglioma cells. Cell Mol Neurobiol. 2015;35(6):891-898. doi:10.1007/s10571-015-0184-8

27. Su Z, Hou XK, Wen QP. Propofol induces apoptosis of epithelial ovarian cancer cells by upregulation of microRNA let-7i expression. Eur J Gynaecol Oncol. 2014;35(6):688-691.

28. Zhu F, Li Q, Yang Y, Wang L, Wang J. Propofol suppresses proliferation, migration, invasion and promotes apoptosis by upregulating microRNA-140-5p in gastric cancer cells. Onco Targets Ther. 2019;12:10129-10138. doi:10.2147/OTT.S225360

29. Yang C, Gao J, Yan N, et al. Propofol inhibits the growth and survival of gastric cancer cells in vitro through the upregulation of ING3. Oncol Rep. 2017;37(1):587-593. doi:10.3892/or.2016.5218

30. Sui H, Zhu C, Li Z, Yang J. Propofol suppresses gastric cancer tumorigenesis by modulating the circular RNA-PVT1/miR-195-5p/ E26 oncogene homolog 1 axis. Oncol Rep. 2020;44(4):1736-1746.

31. Zhao H, Wei H, He J, et al. Propofol disrupts cell carcinogenesis and aerobic glycolysis by regulating circTADA2A/miR-455-3p/FOXM1 axis in lung cancer. Cell Cycle. 2020;19(19):2538-2552. doi:10.1080/ 15384101.2020.1810393

32. Fang Q, Yang A, Dong A, Zhao L. circPDSS1 stimulates the development of colorectal cancer via activating the wnt $/ \beta$-catenin signaling. Onco Targets Ther. 2020;13:6329-6337. doi:10.2147/OTT.S249853 
33. Yu Q, Liu P, Han G, Xue X, Ma D. CircRNA circPDSS1 promotes bladder cancer by down-regulating miR-16. Biosci Rep. 2020;40(1): BSR20191961. doi:10.1042/BSR20191961

34. Hansen TB, Jensen TI, Clausen BH, et al. Natural RNA circles function as efficient microRNA sponges. Nature. 2013;495 (7441):384-388. doi:10.1038/nature11993

35. Chu YL. Circ_0067934 correlates with poor prognosis and promotes laryngeal squamous cell cancer progression by sponging miR-1324. Eur Rev Med Pharmacol Sci. 2020;24(8):4320-4327.

36. Zhu Q, Lu G, Luo Z, et al. CircRNA circ_0067934 promotes tumor growth and metastasis in hepatocellular carcinoma through regulation of miR-1324/FZD5/Wnt/ $\beta$-catenin axis. Biochem Biophys Res Commun. 2018;497(2):626-632. doi:10.1016/j.bbrc.2018.02.119

37. Vervoort SJ, van Boxtel R, Coffer PJ. The role of SRY-related HMG box transcription factor 4 (SOX4) in tumorigenesis and metastasis: friend or foe? Oncogene. 2013;32(29):3397-3409. doi:10.1038/ onc. 2012.506
38. Mehta GA, Parker JS, Silva GO, Hoadley KA, Perou CM, Gatza ML. Amplification of SOX4 promotes PI3K/Akt signaling in human breast cancer. Breast Cancer Res Treat. 2017;162(3):439-450. doi:10.1007/s10549-017-4139-2

39. Zhang P, Li J, Song Y, Wang X. MiR-129-5p inhibits proliferation and invasion of chondrosarcoma cells by regulating $\mathrm{SOX} 4 / \mathrm{Wnt} / \beta-$ catenin signaling pathway. Cell Physiol Biochem. 2017;42 (1):242-253. doi:10.1159/000477323

40. Zhang J, Zhang K, Hou Y. Long non-coding RNA NNT-AS1 knockdown represses the progression of gastric cancer via modulating the miR-142-5p/SOX4/Wnt/ $\beta$-catenin signaling pathway. Mol Med Rep. 2020;22(2):687-696. doi:10.3892/mmr.2020.11158

41. Pang L, Li B, Zheng B, Niu L, Ge L. miR-138 inhibits gastric cancer growth by suppressing SOX4. Oncol Rep. 2017;38(2):1295-1302. doi:10.3892/or.2017.5745

\section{Publish your work in this journal}

Cancer Management and Research is an international, peer-reviewed open access journal focusing on cancer research and the optimal use of preventative and integrated treatment interventions to achieve improved outcomes, enhanced survival and quality of life for the cancer patient.
The manuscript management system is completely online and includes a very quick and fair peer-review system, which is all easy to use. Visit http://www.dovepress.com/testimonials.php to read real quotes from published authors. 